\title{
GINGER - Toward an experimental test of General Relativity
}

\author{
Alberto Porzio* \\ CNR - SPIN and INFN, Naples, Italy \\ E-mail: alberto.porzio@na.infn.it
}

\section{Carlo Altucci}

Dipartimento di Fisica, Università "Federico II" and INFN, Naples, Italy

E-mail: carlo.altucciena.infn.it

\section{Salvatore Capozziello}

Dipartimento di Fisica, Università "Federico II" and INFN, Naples, Italy

E-mail: salvatore.capozziello@na.infn.it

\section{Raffaele Velotta}

Dipartimento di Fisica, Università "Federico II" and INFN, Naples, Italy

E-mail: raffaele.velotta@na.infn.it

\section{Angela Di Virgilio}

INFN Pisa, Italy

E-mail: angela.divirigilio@pi.infn.it

\section{Andrea Basti}

Dipartimento di Fisica, Università di Pisa, Italy

E-mail: andrea.basti@unipi.it

\section{Nicoló Beverini}

Dipartimento di Fisica, Università di Pisa and INFN Pisa, Italy

E-mail: nicolo.beverini@unipi.it

\section{Giorgio Carelli}

Dipartimento di Fisica, Università di Pisa and INFN, Pisa, Italy

E-mail: giorgio.carellieunipi.it

\section{Donatella Ciampini}

Dipartimento di Fisica, Università di Pisa, Italy

E-mail: donatella.ciampinieunipi.it

\section{Francesco Fuso}

Dipartimento di Fisica, Università di Pisa, Italy

E-mail: francesco.fusodunipi.it

\section{Umberto Giacomelli}

Dipartimento di Fisica, Università di Pisa and INFN, Pisa, Italy

E-mail: umberto.giacomelli@pi.infn.it 


\section{Enrico Maccioni}

Dipartimento di Fisica, Università di Pisa and INFN Pisa, Italy

E-mail: enrico.maccionieunipi.it

Fabio Stefani

Dipartimento di Fisica, Università di Pisa and INFN, Pisa, Italy

E-mail: fabio.stefani@unipi.it

\section{Giuseppe Terreni}

INFN, Pisa, Italy

E-mail: giuseppe.terreni@pi.infn.it

\section{Antonello Ortolan}

INFN, Legnaro (PD), Italy

E-mail: antonello.ortolanelnl.infn.it

GINGER (Gyroscopes IN General Relativity) is a proposal for measuring the Lense-Thirring effect using an array of ring laser-gyroscopes. Those are, nowadays, the most sensitive inertial sensors to measure the rotation rate of the Earth. The Lense-Thirring contribution to the Earth gravitational field marks itself as a tiny DC perturbation onto $\Omega$, the Earth rotation rate. Its magnitude is $10^{-9} \times \Omega$ so that to be able to discriminate it a very high sensitivity and long measurement times in order to move toward low frequency are required. For such an experiment, an underground location guarantees further isolation from anthropic as well as environmental disturbances. GINGERINO is a single axis ring laser located inside the the INFN Gran Sasso laboratory. It has demonstrated that the very high thermal stability of the cave allows continuous operation, and sensitivity well below fractions of nrad/s are feasible with duty cycle above $90 \%$ even without stabilisation of the scale factor of the ring laser. Here we show the GINGER experiment concept together with the first evaluation of the GINGERINO sensitiviy that shows how such a device can be of use also in earth science and related phenomena.

Corfu Summer Institute 2017 "School and Workshops on Elementary Particle Physics and Gravity"

2-28 September 2017

Corfu, Greece

${ }^{*}$ Speaker. 


\section{Introduction}

Ring Lasers Gyroscopes (RLG) exploit Sagnac effect to reach high sensitivity in measuring absolute rotation rate $[1,2]$. They are very reliable instruments, with extended bandwidth and very high duty cycle $[3,4,5]$. Their sensitivity increases quadratically with their size. Small size RLGs are used for inertial navigation and mostly all the aircraft and ships carry one RLG on board. So far, the RLG with the highest sensitivity is the Gross Ring $G$ of the geodetic observatory of Wettzell (Germany), a square RLG with a $16 \mathrm{~m}$ perimeter. It has demonstrated that RLG noise level can operate at the shot noise limit reaching the sensitivity of $10^{-13} \mathrm{rad} / \mathrm{s}$ in one day of integration time. The $G$ ring is, presently, used in rotational seismology to measure daily and sub-daily variations of the Earth rotation rate up to the detection of very long period geodetic effects on the Earth rotation vector $[3,6,7]$. This unprecedented sensitivity shows that large RLGs, upon increasing the long term stability of the apparatus in order to improve the integration time, are good candidates for testing General Relativity (GR).

In addition to the well established Sagnac effect RLGs feel all non reciprocal effects affecting the two counter-propagating beams. Following GR the gravito-magnetic and gravito-electric fields are indeed non reciprocal effects [8], so that the measured angular velocity contains, in addition to the kinematic term, additional GR terms that are of the order of $\sim 10^{-13} \mathrm{rad} / \mathrm{s}$, that is very close to the present best sensitivity (in one day of integration time). The purpose of GINGER (Gyroscopes IN GEneral Relativity) is to measure the GR components of the gravito-magnetic field of the Earth $[4,9]$. The GINGER novelty is twofold: a) the sensitivity goal $(1 \%)$ on the measurement of the so called Lense-Thirring effect will be unprecedented; so far, the gravito-magnetic field of the Earth has been measured in space with an error at the $\sim 5 \%[10,11,12,13]$ level; b) it aims at measuring the GR effect of a rotating frame being tied on the Earth itself [14]; GINGER would provide the first measurement of a GR dynamic effect of the gravitational field on the Earth surface. This in turns implies that it would be a direct local measurement, independent of the global distribution of the gravitational field and not an average value, as in the case of space experiments.

The output signal of a RLG is the beat note of the two counter-propagating modes, the frequency of the beat note is called the Sagnac frequency $f_{S}$ and is given by:

$$
f_{s}=\frac{4 \pi A}{P \lambda} \hat{n} \cdot \vec{\Omega}_{T}
$$

where $\hat{n}$ is the area versor of the ring cavity, $A$ the area modulus, $P$ the perimeter and $\lambda$ is the light wavelength. All together these factors give the scalar factor $S=\frac{4 \pi A}{P \lambda}$ of the gyroscope. $S$ is purely geometrical and its knowledge, as we will discuss in the following, is a critical issue for reaching the target sensitivity [15]. $\vec{\Omega}_{T}$ is the angular rotation which affects the RLG cavity. If the RLG is attached to the Earth crust, $\vec{\Omega}_{T}$ is the sum of the angular rotation of the Earth $\vec{\Omega}_{\oplus}$, local disturbances generated by human activity and by geophysical origin $\vec{\Omega}_{L}$, and the GR corrections which are seen locally as angular rotation vectors $\vec{\Omega}_{G R}[8,9]$.

Contrarily to local disturbances and geophysical signals, GR terms are DC quantities. The effect of the gravito-magnetic (Lense-Thirring, LT) and gravito-electric (deSitter, dS) fields is an angular velocity $\Omega_{L T}$ and $\Omega_{d S}$, that combines with $\Omega_{\oplus}$, the Earth angular velocity and GR gives precisely $\Omega_{L T}$ and $\Omega_{d S}$ orientation and amplitudes in function of the latitude of the observer. Note 


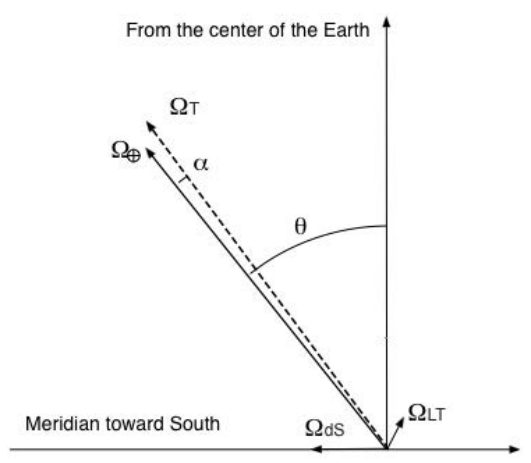

Figure 1: The three axial vectors $\vec{\Omega}_{\oplus}, \vec{\Omega}_{L T}$, and $\vec{\Omega}_{d S}$ are shown, with the relative orientation at the latitude of the underground laboratory of GranSasso (LNGS), following General Relativity. The angle $\alpha$ and $\Omega_{T}$ (dashed line) are shown in the picture. The graph is not on scale, it gives a pictorial view of the relative orientations of the different components, in the reality the modulus of $\vec{\Omega}_{\oplus}$ is 9 orders on magnitude bigger than the GR terms, and the angle $\alpha$ is of the order of $\sim 3.5 \cdot 10^{-10} \mathrm{rad}$ at the latitude of $45^{\circ}$.

also that several alternative theories of gravitation predict different dependences on latitude; so that a direct observation would, perhaps, discriminate between different theories. Figure 1 shows the mutual orientation of the various vectors for an assumed location of $\simeq 45^{\circ}$ latitude (as e.g. at the Gran Sasso laboratories, LNGS) as given by GR.

The purpose of GINGER is to measure the GR components of the gravitational field of the Earth at $1 \%$, using an array of ring-lasers $[9,4,14]$.

We have recently studied properties of an array composed of two RLGs [14], one oriented at the maximum of the signal and the other laying horizontally. In that paper we defined all the constrains on the set-up in order to reach the $1 \%$ goal for the Lense-Thirring test. The RLG at the maximum signal provides an excellent measurement of the modulus of the total rotation rate, while the combination with the horizontal one provides redundancy of measurements and a good measurement of the polar motion. In the following we briefly review the GINGER experimental principles and give some results of the actual operating prototype (called GINGERINO) installed at LNGS. In particular, we will give a glance onto the connection between geophysics and GINGERINO.

\section{The GINGER array}

As already shown, the Sagnac frequency $f_{S}$ of the RLG is proportional to the flux of the total rotation vector $\vec{\Omega}_{T}$ across the area of the ring through the so called scale factor $S$ (see Eq. 1.1). Taking into account the fact that $\Omega_{\oplus}$ and the GR terms are contained inside the meridian plane, it is possible to restrict the analysis to the case of only two RLGs, arranged in such a way that the relative area versors $\hat{n}$ and the $\vec{\Omega}_{T}$ are all contained in the meridian plane, and the problem becomes bi-dimensional. Labelling the two rings as $\mathrm{RLG}_{2}$ and $\mathrm{RLG}_{1}$ and making the scalar products explicit in the relative Sagnac frequency expression, we may write:

$$
f_{S_{1}}=S_{1} \Omega_{T} \cos (\gamma-\zeta)
$$




$$
f_{S_{2}}=S_{2} \Omega_{T} \cos \zeta
$$

where $\zeta$ is the angle between $\vec{\Omega}_{T}$ and $\hat{n}_{2}$, while $\gamma$ is the angle between $\hat{n}_{2}$ and $\hat{n}_{1}$, the area versors of the two RLGs respectively, which we assume can be independently measured. The two indices can be interchanged. Assuming equal scale factors $\left(S_{2}=S_{1}=S\right.$ ) and solving Eq. (2.1) $\Omega_{T}$ (the modulus of $\vec{\Omega}_{T}$ ), and $\zeta$ can be retrieved:

$$
\begin{aligned}
\zeta & =\tan ^{-1} \frac{f_{1}-f_{2} \cos (\gamma)}{f_{2} \sin (\gamma)} \\
\Omega_{T} & =\frac{\sqrt{f_{1}^{2}+f_{2}^{2}-2 f_{1} f_{2} \cos (\gamma)}}{S \sin (\gamma)}
\end{aligned}
$$

The combination of the frequencies of the two RLGs allows measuring the amplitude of $\Omega_{T}$, and, if the angle $\gamma$ between the two RLGs is independently known, it is possible to retrieve $\zeta$, the angle between the versor $\hat{n}_{2}$ and the axis of the vector $\vec{\Omega}_{T}$. The combination of two RLGs with one oriented along the rotation axis, therefore at the maximum of the signal, allows very high precision in the determination of the angle $\zeta$, and the measurement of $\Omega_{T}$, as it will be shown in the following.

It goes without saying that this ideal situation contrasts with the real apparatus for which it is necessary to estimate the error in the measurement of $\zeta$, which depends on the independent measurement of $\gamma$ (the angle between the two RLGs) and the indetermination of the frequencies, i.e. the shot noise. A detailed discussion on the error budget and the constraints on the apparatus has been discussed in Ref. [14].

GINGER will be attached to the Earth crust: the mirrors will follow the crust motion and the RLGs will be sensitive to $\vec{\Omega}_{\oplus}$, the Earth rotation rate, plus other terms, for example the GR corrections, tides and terms originated by global or local phenomena. Neglecting all the local contributions but the GR corrections, let's call $\vec{\omega}$ the sum of the GR terms affecting the apparatus so that $\vec{\Omega}_{T}=\vec{\Omega}_{\oplus}+\vec{\omega}$. With respect to $\vec{\Omega}_{\oplus}, \vec{\Omega}_{T}$ will show different amplitude and direction. Applying the distributive property of the scalar product, Eqs. (2.1) become:

$$
\begin{aligned}
& f_{1}=S \times\left(\Omega_{\oplus} \cos (\gamma-\zeta+\alpha)+\omega \cos (\xi-(\gamma-\zeta+\alpha))\right. \\
& f_{2}=S \times\left(\Omega_{\oplus} \cos (\zeta-\alpha)+\omega \cos (\xi+\zeta-\alpha)\right)
\end{aligned}
$$

where $\xi$ is the angle between $\vec{\Omega}_{\oplus}$ and $\vec{\omega}$ while $\alpha$ is the one between $\vec{\Omega}_{\oplus}$ and $\vec{\Omega}_{T}$. It is necessary to note that if $\mathrm{RLG}_{2}$ is horizontal, the colatitude $\theta$ is $\theta=\zeta-\alpha$.

Both theory and already in operation RLGs have proven that $\vec{\Omega}_{\oplus}$ is several orders of magnitude higher than any correction (GR and local ones). Accordingly, we can write $\vec{\omega}$ as $|\vec{\omega}|=\omega=\eta \Omega_{\oplus}$ with $\eta \ll 1$. Here we consider the simplest case considering as given the angle $\xi$ between $\vec{\Omega}_{\oplus}$ and $\vec{\omega}$, with the more general case already discussed in [14]. As above, we consider RLG $\mathrm{R}_{1}$ aligned along $\vec{\Omega}_{T}$ so that $\zeta_{1} \ll 1$ and $\zeta_{1}=\gamma-\zeta$ (angle between $\hat{n}_{1}$ and $\vec{\Omega}_{T}$ ). Substituting in Eqs. 2.3 we obtain:

$$
\begin{aligned}
& f_{1}=S \Omega_{\oplus} \times\left(\cos \left(\zeta_{1}+\alpha\right)+\eta \cos \left(\xi-\left(\zeta_{1}+\alpha\right)\right)\right. \\
& f_{2}=S \Omega_{\oplus} \times(\cos (\zeta-\alpha)+\eta \cos (\xi+\zeta-\alpha))
\end{aligned}
$$

Asking that the derivative in $\zeta$ of frequency $f_{1}$ equals 0 for $\zeta=0$, i.e. requiring that $\mathrm{RLG}_{1}$ is aligned along $\Omega_{T}$, and solving for $\alpha$, at first order in $\eta$ we find:

$$
\alpha=\eta \sin (\xi)
$$


Then expanding at the first order in $\alpha, \zeta_{1}$ and $\eta$ we have:

$$
\begin{aligned}
& f_{1}=S \Omega_{\oplus}(1+\eta \cos \xi) \\
& f_{2}=S \Omega_{\oplus} \cos \zeta(1+\eta \cos \xi)
\end{aligned}
$$

The above relations allow to measure $\eta$ (assuming $\cos \xi \neq 0$, and $\cos \zeta \neq 0$ ):

$$
\begin{aligned}
& \eta=\frac{f_{1}-S \Omega_{\oplus}}{S \Omega_{\oplus} \cos \xi} \\
& \eta=\frac{f_{2}-S \Omega_{\oplus} \cos \zeta}{S \Omega_{\oplus} \cos \xi \cos \zeta}
\end{aligned}
$$

The two solutions are equivalent thus, each of the two RLG can give $\eta$. This is a consequence of the fact that we assumed that $\xi$ is known [8]. Accordingly the comparison of the two measurements will provide a check of $\xi$, and it can be used to verify whether the measurement is affected by systematic errors of the ring lasers.

\subsection{The RLG signal expressed with the GR terms}

The GR terms depend on the ratio of Schwarzschild radius to the average radius of the Earth $[8$, App. A]

$$
a \equiv \frac{2 G M_{\oplus}}{R_{\oplus} c^{2}} \simeq 1.3918082245(20) \times 10^{-9}
$$

and on the Earth dimensionless momentum of inertia

$$
b \equiv \frac{G I_{\oplus}}{R_{\oplus}^{3} c^{2}} \simeq 2.301326(700) \times 10^{-10}
$$

, taking this into account each RLG output frequency $f$ can be expressed in terms of $a$, and $b$ as:

$$
f=S \Omega_{\oplus}|\cos (\zeta-\alpha)-(a-b) \sin \theta \sin (\zeta-\alpha-\theta)+2 b \cos \theta \cos (\zeta-\alpha-\theta)|
$$

where the absolute value bars $\mid$ have been introduced just to remember that the frequency is always a positive quantity. The angle $\alpha$ is well defined by GR [8] and it is $\alpha=(a-3 \cdot b) \cos \theta \sin \theta$, and considering that $\alpha=\eta \sin (\xi)$ (see Eq. (2.5)) it is possible to evaluate $a$ and $b$ once $\eta$ is measured.

From the numerical value of $a$ and $b$ (see above) it is possible to show that considering all the GR contributions it is $\eta \sim 8.82 \cdot 10^{-10}$ and $\xi \sim 23.37^{\circ}$. Isolating the LT effect from the other GR contributions one would get $\eta \sim 3.64 \cdot 10^{-10}$ and $\xi \sim 71.56^{\circ}$.

\section{Earth science and the LNGS prototype GINGERINO}

The Earth is a solid body, as a consequence each crustal perturbation, like earthquakes, locally generates translations and rotations. The use of rotational sensors in addition to linear seismometers, that equipped standard seismic stations, would give the possibility to reconstruct the direction of propagation of the seismic waves and their phase velocity. Moreover, any tectonic motion causes a local rotation with respect to the center of the Earth. In principle extracting these signals from RLGs could be an independent way to measure tectonic plates motions. 


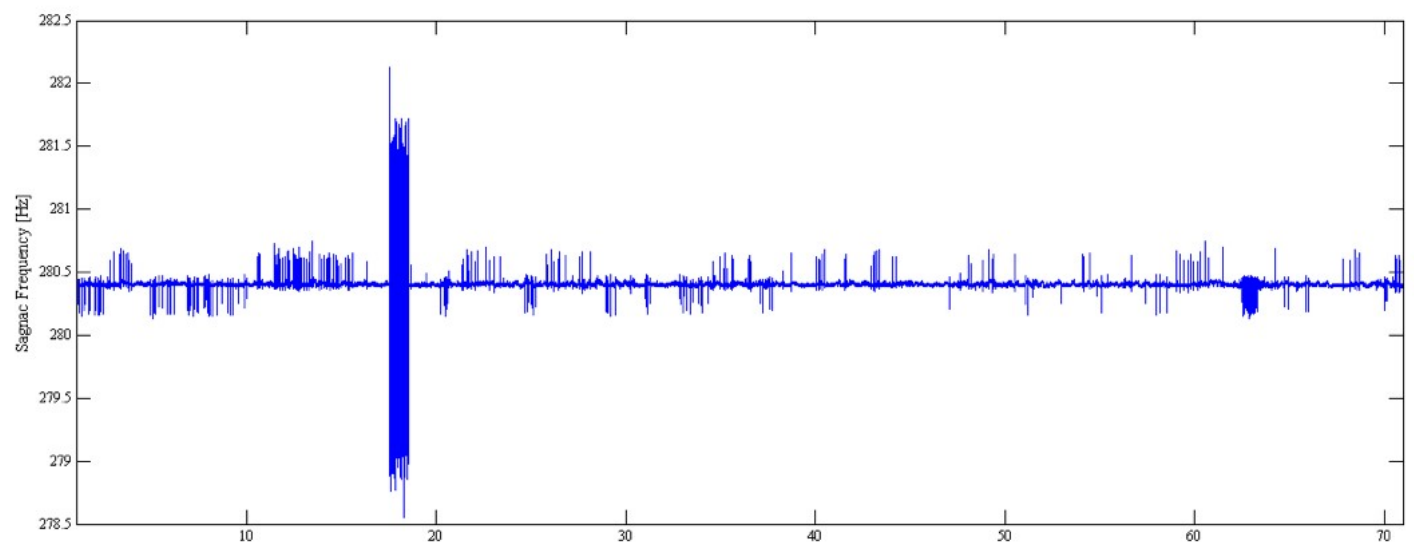

Figure 2: Sagnac frequency measured during 76 days of the uninterrupted operation of GINGERINO starting on August 2017. The frequency is centered around the $\simeq 280 \mathrm{~Hz}$ value in agreement with the design of the RLG. Spikes over short period represent typical transient signals coming from tele-seismic events.

RLGs can monitor polar motion and the Earth rotation rate changes with high rate so giving insights also in geodesy. Presently, they are considered the only instruments which can provide accurate measurements of the orientation change of the Earth axis in the time scale of the length of the day (LOD) [16].

While GR contributions are stationary so that they produce DC, indeed tiny, shift of the Earth rotation Sagnac frequency, all these geo-linked signals are transient or periodic and can be seen as perturbation temporally confined in RLG traces.

It goes without saying that the detectability of such signals strongly depends on the sensitivity and the bandwidth of the instrument. Most of these signals are, in any case, low frequency ones, from a few $\mathrm{Hz}$ down to less than one cycle per day and even longer. In principle, the RLG is not limited from below in the bandwidth, while it may appear some technical limitation on the apparatus response and on the reconstruction of the signal.

GINGERINO is a square RLG with a side of $3.6 \mathrm{~m}$ developed after the first INFN prototype GPisa in operation since 2009 [18]. It is running inside the deep underground INFN laboratory of the Gran Sasso (LNGS) [5, 17]. It aims to characterise the underground rotational seismic and anthropic disturbances so to validate the site in view of the GINGER apparatus. Settled at the end of 2014, began data taking in spring 2015. Despite the fact that this device is an R\&D prototype it has run continuously for months without any active control on the cavity geometry (perimeter and/or side lengths), thanks to the very quiet and thermally stable environment at LNGS.

During 2017, GINGERINO has been in continuous, uninterrupted and unattended operation for a long period with a very large duty-cycle of the measurements (above $95 \%$ of the total operation time).

Figure 2 shows the raw data for the Sagnac frequency measured in 76 days period.

It has to be noted that the low frequency part of the noise spectrum is dominated by backscattering that represents the unwanted residual coupling between the two counter-propagating beams through residual scattering processes at the mirror surfaces [19]. This effect, for a larger part, can be off-line subtracted and, in GINGER would be mitigated by both the larger size (so that the solid 


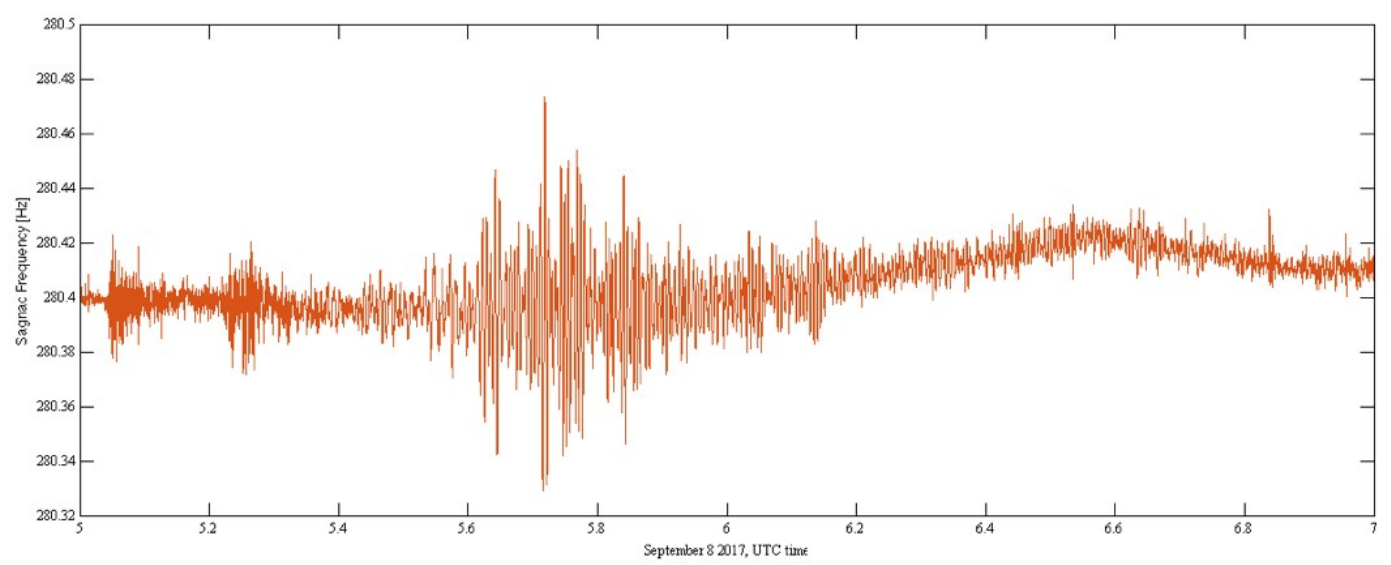

Figure 3: Variations of the angular velocity measured during a tele-seismic (M 8.0 earthquake, near the coast of Chiapas, Mexico August 8, 2017).

angle of the mode would be strongly reduced) and an improved mirror quality.

Having a data set extending over a long period is a demonstration of the long term stability of the apparatus, which cannot be otherwise ascertained. Moreover, such a long term stability makes GINGERINO a sensor that can look at Earth rotational motion in the ultra-low frequency range.

As already stated, RLG represents an extremely precious tool for seismology. Moreover, the underground location at LNGS and the occurrence of frequent seismic events in a radius of few hundreds km, make GINGERINO a unique seismological observatory, potentially able to provide relevant insight onto geophysics and geodesic issues. As already demonstrated in the past, GINGERINO is in fact sensitive on both tele-seismic and local seismic events: Fig. 3 shows a representative data set. In this plot the transient effect on the Sagnac frequency of a far away very strong earthquake is reported.

The long-term uninterrupted operation enables analyzing the behavior of GINGERINO at the very low frequency, where geodetic phenomena (e.g., polar motion, solid tides) of very large relevance occur on different time scales. The analysis of the Allan deviation calculated over raw and duly manipulated data [17] for a 90 days data set shows that GINGERINO has reached the sensitivity of the order of $\approx \mathrm{prad} / \mathrm{s}$ with one day of integration. We note that a factor 20 improvement in the sensitivity would make the device sensitive to geodesic effects, such as polar motion and solid Earth tides.

In the short time scale the actual noise floor is less than one order of magnitude larger than the quantum noise [17]. On the contrary, on the long time scale the deviation is more pronounced, as expected because of the presence of various environmental and technical disturbances. In particular, we expect that the very recent installation of higher quality mirrors at LNGS would improve this part of the spectrum.

The quantum limit of such a detector depends in turn on the laser power (higher power implies lower quantum limit for the detector sensitivity) and size of the ring (lower noise for larger ring). While the operating power is not a tuning knob of the apparatus because for stability reason the laser needs to operate close to its threshold, GINGER would be substantially larger than GINGERINO so that its actual limit would be lower. Moreover, the implementation, in GINGER, of active controls 
on the RLG geometry [15] would surely improve the long term stability of the scale factor.

\section{Conclusions and perspectives}

The GINGER project aims to measure GR effects on the Earth rotation by means of an array of RLGs. The Lense-Thirring gravito-magnetic effect represents a unique signature of general relativity onto the stationary rotation rate of the Earth. It manifests itself as tiny variations of both amplitude and direction of the Earth rotation vector. The possibility of measuring such a tiny effect resides in the precision of large (several squared meters) RLGs: the best performing rotation sensors actually in operation. We have discussed the principles of this challenging and fascinating measure and presented the performances of a RLG prototype, GINGERINO, actually in data taking at LNGS. A configuration made of two RLGs in the meridian plane, one oriented along the Earth rotation axes (so at the maximum of the signal), the other at a given relative angle $\gamma$, reduces the three-dimensional problem into a two-dimensional one and would allow, under some technical and geometrical constraints, to improve measurement precision up to the level required for a fully reliable detection of GR effects. GINGERINO, at present time running inside LNGS, has reached a twofold goal. On one hand, it has proven that LNGS environment is sufficiently far from anthropic and surface disturbances thus giving an outstanding sensitivity and ability of continuous operation. On the other hand, it has reached a sensitivity that allows the use of its data for seismic studies.

GINGER is thought to be an interdisciplinary unique observatory where the Earth could be studied under very different perspectives: the closest GR field relevant source and the unknown strange solid object we live on.

\section{References}

[1] G. Sagnac, L'éther lumineux démontré par l'effet du vent relatif d'éther dans un interférométre en rotation uniforme [The demonstration of the luminiferous aether by an interferometer in uniform rotation], Comptes Rendus 157:708 (1913); G. Sagnac Sur la preuve de la réalité de l'éther lumineux par l'expérience de l'interférographe tournant [On the proof of the reality of the luminiferous aether by the experiment with a rotating interferometer], Comptes Rendus 157:1410 (1913);

[2] The Sagnac effect: 100 years later /L'effet Sagnac : 100 ans aprés, Special Issue in Comptes Rendus Physique 15, pp. 787-916, Gauguet Alexandre editor (2014);

[3] K.U. Schreiber and J.P. Wells, Large ring lasers for rotation sensing, Rev. of Scient. Instrum. 84:041101 (2013);

[4] A. Di Virgilio, M. Allegrini, A. Beghi, J. Belfi, N. Beverini, F. Bosi, B. Bouhadef, M. Calamai, G. Carelli, D. Cuccato, E. Maccioni, A. Ortolan, G. Passeggio, A. Porzio, M. L. Ruggiero, R. Santagata, A. Tartaglia, A ring lasers array for fundamental physics, Comptes Rendu Physique 15:866 (2014); N. Beverini, M. Allegrini, A. Beghi, J. Belfi, B. Bouhadef, M. Calamai, G. Carelli, D. Cuccato, A. Di Virgilio, E. Maccioni, A. Ortolan, A. Porzio, R. Santagata, S. Solimeno and A. Tartaglia, Measuring general relativity effects in a terrestrial lab by means of laser gyroscopes, Las. Phys. 24:074005 (2014);

[5] J. Belfi, N. Beverini, F. Bosi, G. Carelli, D. Cuccato, G. De Luca, A. Di Virgilio, A. Gebauer, E. Maccioni, A. Ortolan, A. Porzio, G. Saccorotti, A. Simonelli, and G. Terreni, Deep underground rotation measurements: GINGERino ring laser gyroscope in Gran Sasso, Rev. of Scient. Instrum. 88:034502 (2017); 
[6] K. U. Schreiber, T. Klügel, J.-P. R. Wells, R. B. Hurst, and A. Gebauer, How to detect the chandler and the annual wobble of the earth with a large ring laser gyroscope, Phys. Rev. Lett. 107:173904 (2011);

[7] C. Hadziioannou, P. Gaebler, U. Schreiber, J. Wassermann, and H. Igel, Examining ambient noise using colocated measurements of rotational and translational motion, J. Seismol. 16:787 (2012);

[8] A. Tartaglia, A. Di Virgilio, J. Belfi, N. Beverini and M.L. Ruggiero, Testing general relativity by means of ring lasersâĂŔ, Eur. Phys. J. Plus 132:73 (2017);

[9] F. Bosi, G. Cella, A. Di Virgilio, A. Ortolan, A. Porzio, S. Solimeno, M. Cerdonio and J. P. Zendri, M. Allegrini, J. Belfi, N. Beverini, B. Bouhade G. Carelli, I. Ferrante, E. Maccioni, R. Passaquieti, F. Stefani, M. L. Ruggieror A. Tartaglia, K. U. Schreibert, A. Gebaueru, and J-P. R. Wells, Measuring gravitomagnetic effects by a multi-ring-laser gyroscope, Phys. Rev. D 84:122002 (2011);

[10] C. W. F. Everitt, D. B. DeBra, B. W. Parkinson, J. P. Turneaure, J. W. Conklin, M. I. Heifetz, G. M. Keiser, A. S. Silbergleit, T. Holmes, J. Kolodziejczak, M. Al-Meshari, J. C. Mester, B. Muhlfelder, V. G. Solomonik, K. Stahl, P. W. Worden Jr., W. Bencze, S. Buchman, B. Clarke, A. Al-Jadaan, H. Al-Jibreen, J. Li, J. A. Lipa, J. M. Lockhart, B. Al-Suwaidan, M. Taber, and S. Wang, Gravity Probe B: Final Results of a Space Experiment to Test General Relativity, Phys. Rev. Lett., 106:221101 (2011);

[11] D. M. Lucchesi, L. Anselmo, M. Bassan, C. Pardini, R. Peron, G. Pucacco, and M. Visco, Testing the gravitational interaction in the field of the Earth via satellite laser ranging and the Laser Ranged Satellites Experiment (LARASE), Class. Quantum Grav. 32:155012, (2015);

[12] I. Ciufolini, A. Paolozzi, E. C. Pavlis, R. König, J. Ries, V. Gurzadyan, R. Matzner, R. Penrose, G. Sindoni, C. Paris, H. Khachatryan, S. Mirzoyan, A test of general relativity using the LARES and LAGEOS satellites and a GRACE Earth gravity model, Eur. Phys. J. C 76:120, (2016);

[13] S. Capozziello, G. Lambiase, M. Sakellariadou and A. Stabile, Constraining models of extended gravity using Gravity Probe B and LARES experiments, Phys. Rev. D 91:044012 (2015);

[14] A. D. V. Di Virgilio, J. Belfi, W.-T. Ni, N. Beverini, G. Carelli, E. Maccioni, and A. Porzio, GINGER: a feasibility study, Eur. Phys. J. Plus 132:157 (2017);

[15] R. Santagata, A. Beghi, J. Belfi, N. Beverini, D. Cuccato, A. Di Virgilio, A. Ortolan, A. Porzio, and S. Solimeno, Optimization of the geometrical stability in square ring laser gyroscopes, Class. Quantum Grav. 32:055013 (2015);

[16] K. U. Schreiber,T. Klügel, A. Velikoseltsev, W. Schlüter, G. E. Stedman, J.-P. R. Wells, The Large Ring Laser G for Continuous Earth Rotation Monitoring, Pure and Applied Geophysics 166:1485 (2009);

[17] J. Belfi, N. Beverini, G. Carelli, A. Di Virgilio, U. Giacomelli, E. Maccioni, A. Simonelli, F. Stefani, G. Terreni, Analysis of 90 days operation of the gyroscope GINGERINO, arXiv:1804.02569 (2018);

[18] A. Di Virgilio, M. Allegrini, J. Belfi, N. Beverini, F. Bosi, G. Carelli, E. Maccioni, M. Pizzocaro, A. Porzio, U. Schreiber, S. Solimeno, and F. Sorrentino, Performances of ${ }^{\prime}$ G-Pisa' : a middle size gyrolaser, Class. Quantum Grav. 27:084033 (2010);

[19] A. Beghi, J. Belfi, N. Beverini, B. Bouhadef, D. Cuccato, A. Di Virgilio, A. Ortolan, Compensation of the laser parameters fluctuations in large ring laser gyros: a Kalman filter approach, Appl. Opt. 51:7518 (2012). 